\title{
Echo Modeling and Simulation Analysis of Millimeter Wave Proximity Fuze ${ }^{1}$
}

\author{
Jie Zhou, Quanli Ning, Chunsheng Hu \\ Army Officer Academy,Hefei,Anhui,230031,China \\ 962854399@qq.com
}

Keywords: millimeter wave proximity fuze, echo modeling, scattering near zone.

Abstract. This Start research on the scattering mechanism of ground object target, the scattering of the far zone and the near region is defined, and the scattering characteristics of the target is analyzed.Using the distance-Doppler method to divide the fuze beam radiation area, the fuze echo model is established and the model is simulated.The simulation results show that when the millimeter wave fuze is under the different targets, the echo amplitude has different degrees of fluctuation, which is consistent with the result of the multiple documents, proves the correctness of the model.

\section{Introduction}

The surface of the earth was covered by a lot of ground, the formation of the different types of surface environment, such as grassland, sand, cement. When the millimeter wave proximity fuze is implemented to ground attack, different ground conditions have great influence on the performance of the fuze, especially in the measurement of the distance from the surface. Therefore, in order to improve the accuracy of the fuze distance measurement, and to make sure that the fuze can be detonated in an effective distance, the echo model of the millimeter wave proximity fuze is studied.

\section{Analysis of the influence of the near zone scattering characteristics on the target echo of ground objects}

A.The partition of far zone and near zone scattering of fuze

If the length of Fuze antenna is 1 , the length of target is $l^{\prime}$,the distance between the fuze and the target is $R$, the relative position relationship is shown in Figure 1.

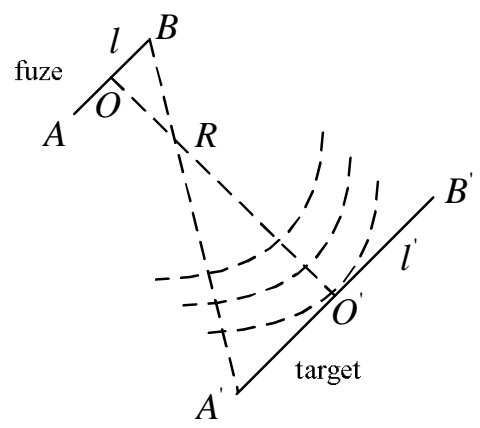

Fig. 1 Sketch Map of electromagnetic interaction between fuze and target

The maximum phase difference of the incident wave is

$$
k_{0}\left(B A^{\prime}-O O^{\prime}\right)=k_{0}\left[\sqrt{R^{2}+\left(\frac{l}{2}+\frac{l^{\prime}}{2}\right)^{2}}-r\right]
$$

Assuming the maximum phase error is $\frac{\pi}{8}$, then

$$
k_{0}\left[\sqrt{R^{2}+\left(\frac{l}{2}+\frac{l^{\prime}}{2}\right)^{2}}-R\right]=\frac{\pi}{8}
$$


When the target size is much larger than the fuze antenna size, it can be further simplified.

$$
R_{\min } \approx \frac{2 l^{2}}{\lambda}
$$

In the formula, $l$ is the maximum size of the target, $\lambda$ is the wavelength of the fuze. When $R>R_{\min }$, the target is called the far zone of the fuze beam. When $R \leq R_{\min }$, the target is called the near zone of the fuze beam.

B.Analysis of the near target scattering characteristics on fuze

It is obvious that the direct application of the far zone scattering theory is not able to solve the problem, but it can be used to divide the target area into the far zone scattering theory.

The target radar cross section is defined in the far zone, and the theoretical formula is as follows:

$$
\sigma_{k}=\lim _{R \rightarrow \infty} 4 \pi R^{2} \frac{\left|H^{s}\right|^{2}}{\left|H^{i}\right|^{2}}=\lim _{R \rightarrow \infty} 4 \pi R^{2} \frac{\left|E^{s}\right|^{2}}{\left|E^{i}\right|^{2}}
$$

The formula (4) is treated by the electromagnetic field vector property:

$$
\sigma_{k}=\frac{\left(\lambda e_{r}^{r} \cdot S\right)^{2}}{\pi}=\frac{\left(\lambda h_{r} \cdot S\right)^{2}}{\pi}
$$

In the formula, $\lambda$ is the wavelength of the fuze, $\dot{e}_{r} 、 \dot{h}_{r}$ is the polarization direction of fuze receiver ,$\stackrel{\prime}{S}$ is the vector scattering function :

$$
\stackrel{r}{S}=k_{0} \operatorname{Re} x p\left(-j k_{0} R\right) \frac{\stackrel{\mathrm{E}}{E}^{S}}{\left|E^{i}\right|}=k_{0} \operatorname{Re} \operatorname{xp}\left(-j k_{0} R\right) \frac{\stackrel{1}{H}^{S}}{\left|H^{i}\right|}
$$

Near zone RCS can be expressed as:

$$
\sigma=\left|\sum_{k=1}^{k=M} \sqrt{\sigma_{k}} \exp \left(\frac{4 \pi R_{k}}{\lambda}\right)\right|^{2}
$$

In the formula, $\sigma_{k}$ is the radar cross section of each scattering plane, $\mathrm{M}$ is the total number of scattered surface elements after target partition, $R_{k}$ is the distance between the fuze and the $\mathrm{k}$ scattering surface element.

\section{Analysis of fuze beam radiation area}

\section{A.Division of beam radiation area of fuze}

At present, there are two main ways of the radiation area dividing method: the distance Doppler division method and the distance division method. In view of the relative motion between the fuze and target, it is necessary to produce the Doppler effect. Therefore, this paper uses the distance Doppler division method.

The core idea of the distance Doppler division method is to divide the distance around the center as the basis, and the vertical line is divided into two kinds of lines, the division of the method is shown in Fig 2.

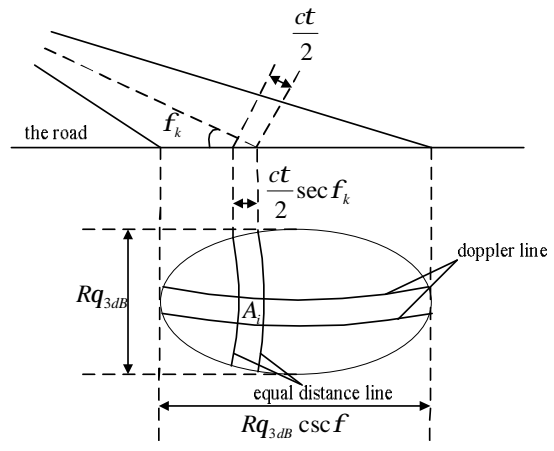

Fig. 2 The schematic diagram of the distance Doppler method 


\section{( 1 ) Lateral spacing}

Transform the formula (3):

$$
R_{\min } \geq \frac{2 l^{2}}{\lambda}
$$

Can be obtained:

$$
\begin{gathered}
l^{\prime} \leq \sqrt{\frac{\lambda R_{\min }}{2}} \\
\Delta R \leq \sqrt{\frac{\lambda R_{\min }}{2}}
\end{gathered}
$$

( 2 ) Longitudinal spacing

All scattered points are required to meet the requirements of the following angular resolution:

$$
\Delta \theta \leq \frac{\lambda}{2 V_{R} \cos \alpha \sin \theta_{\max }} \Delta f
$$

Thus, the number of the scattered body in a distance ring is:

$$
N_{\text {纵向 }}=\frac{\theta_{C}}{\Delta \theta} \geq \frac{2 V_{R} \cos \alpha \sin \theta_{\text {max }}}{\lambda f_{r}} \times N_{F F T} \times \theta_{C}
$$

In the formula, $\theta_{C}$ is the projection angle width of fuze beam in horizontal plane , $N_{F F T}$ is Fu Liye transform length.

\section{Instantaneous echo power of a single surface element}

According to the radar range equation, the instantaneous echo power of a single surface element can be obtained:

$$
P_{r i}\left(\theta_{i}, t\right)=P_{t}\left(t-\tau_{i}(t)\right) \frac{G^{2}\left(\theta_{i}\right) \lambda^{2} \sigma_{i}}{(4 \pi)^{3} R_{i}^{4} L}
$$

In the formula, $\theta_{i}$ is the the azimuth angle of the beam center of the I unit deviated from the center of the beam, $P_{t}$ is the peak transmit power of fuze, $\mathrm{G}$ is the fuze antenna gain, $\tau_{i}$ is the the I units of the fuze and round-trip delay time, $\sigma_{i}$ is the the radar cross section of a unit surface of $\mathrm{I}, R_{i}$ is the distance between the fuze and the I cell surface, $\mathrm{L}$ is the total loss.

$$
\tau_{i}=\frac{2 R_{i}(t)}{c}=\frac{2\left(R_{0}-v_{r i} t\right)}{c}
$$

Can be obtained:

$$
P_{r i}\left(\theta_{i}, t\right)=P_{t}\left[t-\frac{2\left(R_{0}-v_{r i} t\right)}{c}\right] \frac{G^{2}\left(\theta_{i}\right) \lambda^{2} \sigma_{i}}{(4 \pi)^{3} R_{i}^{4} L}
$$

\section{Echo model and simulation analysis of ground objects}

\section{A.Target echo model}

The echo signal received by the fuze is generated by a large number of randomly distributed scattered bodies in the scattering region of the target area.

Assuming the fuze's transmit signal $s(t)$ is :

$$
s(t)=A \sin (\omega t+\varphi)
$$


In the formula, $A$ is the amplitude of transmit signal, $\omega$ is the angular frequency of transmitted signal, $\varphi$ is the initial phase of launch signal.The received signal can be expressed as :

$$
s_{r}(t)=\sum_{i=1}^{M} A_{i} \sin \left(\omega_{i} t+\varphi\right)
$$

In the formula, $\mathrm{M}$ is the number of units after dividing, $A_{i}$ is the signal amplitude of unit $\mathrm{I}, \omega_{i}$ is the signal angular frequency of unit I.

$$
s_{r}(t)=\sum_{i=1}^{M} P_{t}\left[t-\frac{2\left(R_{0}-v_{r} t\right)}{c}\right] \frac{G^{2}\left(\theta_{i}\right) \lambda^{2} \sigma_{i} \sin \left(\omega_{i} t+\varphi\right)}{(4 \pi)^{3} R_{i}^{4} L}
$$

In the formula, $f$ is the fuze firing frequency, $\tau_{i}$ is the two-way time fuze signal by the I unit plane return.

$$
s_{r}(t)=\sum_{i=1}^{M} P_{t}\left[t-\frac{2\left(R_{0}-v_{r} t\right)}{c}\right] \frac{G^{2}\left(\theta_{i}\right) \lambda^{2} \sigma_{i} \sin \left(2 \pi f t+\frac{4 \pi f R_{i}}{c}+\varphi\right)}{(4 \pi)^{3} R_{i}^{4} L}
$$

\section{B.Target echo simulation}

The echo from the surface of soil and rock surface, woods are simulated,Simulation results are shown in fig 3.

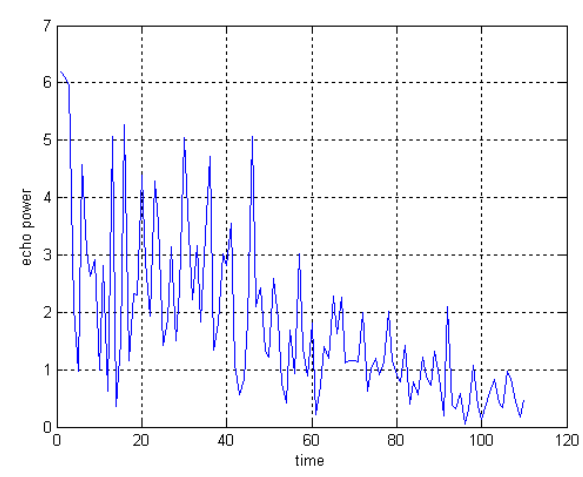

( a ) Soil and rock surface

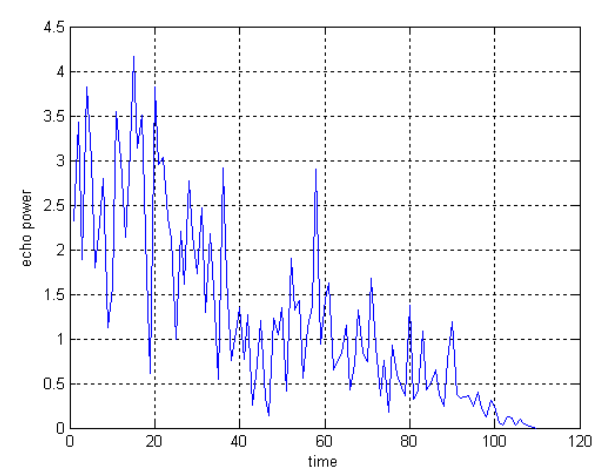

( b ) Woods

C.Simulation result analysis

Fig. 3 simulation results of different targets echo

From the simulation results, it can be seen that the envelope of the echo signal is random fluctuation and the phase is also random fluctuation in the process of fuze to the target. In contrast, the change of the surface of the road is the biggest, which is also verified by the case of the front, the road surface with specular reflection, reflective intensity changes.

\section{Conclusion}

In this paper, the echo model of the millimeter wave proximity fuze is established by using the near zone scattering theory, the model can be used to establish the model simulation environment of fuze target,providing an important reference basis for the simulation test of the fuze development process.

\section{References}

[1] Li Yuehua, Li Xingguo. Beijing: Beijing Institute of Technology press, 2009.12

[2] Cui Zhanzhong, Song Shihe, Xu Lixin. Principle of proximity fuze (Third Edition) [M]. Beijing:

Beijing Institute of Technology press, 2009.08

[3] Zhao Huichang. Design principle and method of radio fuze [M]. Beijing: National Defence Industry Press, 2012.03 
[4] Chen Xiaolu. Research on Key Technologies of low altitude radio fuze [D]. national defense science and Technology University, 2007

[5] Wu Da Kui. Millimeter wave fuze in the research on key technology and application of [D]. Electronic Science and Technology University doctoral dissertation, 2010 